\title{
Can the Standard Model Predict a Minimum Acceleration That Gets Rid of Dark Matter?
}

\author{
Espen Gaarder Haug \\ Norwegian University of Life Sciences, Ås, Norway \\ Email: espenhaug@mac.com
}

How to cite this paper: Haug, E.G. (2021) Can the Standard Model Predict a Minimum Acceleration That Gets Rid of Dark Matter? Journal of High Energy Physics, Gravitation and Cosmology, 7, 602-606. https://doi.org/10.4236/jhepgc.2021.72035

Received: March 7, 2021

Accepted: April 22, 2021

Published: April 25, 2021

Copyright (อ 2021 by author(s) and Scientific Research Publishing Inc. This work is licensed under the Creative Commons Attribution International License (CC BY 4.0).

http://creativecommons.org/licenses/by/4.0/ (c) (i) Open Access

\begin{abstract}
The standard model is considered to be very bad at predicting galaxy rotation, and this is why the hypothesis of dark matter was introduced in physics in the 20th century. However, in this paper, we show that the standard model may not be as far off as previously believed. By taking into account that gravity has an "infinite" extent in space and assessing the assumed mass in the observable universe, we obtain a minimum acceleration that gives a much closer match to observed galaxy rotations than would be expected. We will discuss whether or not this is enough to overturn the long-standing perspective on the standard model and if it could indeed provide a possible and adequate explanation of galaxy rotations.
\end{abstract}

\section{Keywords}

Galaxy Rotation, Dark Energy, Observable Universe, Minimum Acceleration

\section{Introduction}

Newton [1] and Einstein [2] standard gravity model, when based on baryonic matter, give galaxy predictions very different from those actually observed. This is why the hypothesis of dark matter was introduced. As early as the 1880s, Lord Kelvin was describing dark bodies in relation to the Milky Way; Henri Poincare picked up the theme in 1906, actually using the term dark matter in his comments on Kelvin's work. By the 1920s and '30s, the term was gaining interest and a number of astronomers and astrophysicists were exploring its potential. The debate continues today: Could dark matter exist, or could it simply be a fudge factor that enables an incomplete model to fit observations? Modified Newton Dynamics introduced by Milgrom [3] in 1983 suggests a minimum acceleration that is calibrated to the observational data, and the model then fits very well, although from baryonic matter only. However, MOND is more of a curve-fitting 
model since it does not provide a good explanation for why there should be such a minimum acceleration. Here we will also introduce a minimum acceleration, though not by modifying the standard gravity model, but rather by building on its assumption regarding the mass of the observable universe and the radius of the observable universe. It is worth noting that a solution to the dark matter problem can, in principle, also be achieved in the approach of extended gravity (see for example [4]). Extended gravity described by, for example the model described by [5] can be partly seen as a relativistic extension of MOND. Other minimum acceleration models have also been suggested recently. One of these is so-called quantized inertia [6], which according to its inventor, McCulloch, has the advantage of having an explanatory model behind the minimum acceleration, something that seems to be missing in the standard MOND model. The hypothesis that will be suggested here falls into the category of non-relativistic minimum acceleration theories, which in this case also explains the minimum acceleration, unlike the original MOND theory.

The radius of the observable universe, as suggested by standard physics, is approximately $4.4 \times 10^{26}$ meters (93 billion light years), see [7] [8]. The age of the universe is considered to be about 13.77 billion years. In this time period, light can travel $13.77 \times 10^{9} \times c \times 365 \times 24 \times 60 \times 60 \approx 1.3 \times 10^{26}$ meter, this is approximately equal to $\frac{c}{H_{o}}$, where $H_{o}$ is the Hubble constant. The reason the radius of the universe is assumed to be considerably larger than this is due to the assumption of expanding space (inflation). In this paper, we will take that for granted, although that too is a subject of considerable debate. Further, the mass of the observable universe is assumed to be approximately $1.5 \times 10^{53} \mathrm{~kg}$. The mass of the observable universe can be calculated as $M_{u}=\frac{c^{3}}{G H_{o}}$ as shown for example by [9] [10] [11], so there is considerable uncertainty in the exact value here as there is considerable uncertainty in the Hubble constant, and also in $G$. Based on the assumed radius of the universe and the mass of the universe, the minimum gravitational acceleration ${ }^{1}$ of the universe must then be

$$
g_{\text {min }}=\frac{G M_{u}}{r_{u}^{2}} \approx \frac{G \times 1.5 \times 10^{53}}{\left(4.4 \times 10^{26}\right)^{2}} \approx 5.18 \times 10^{-11} \mathrm{~m} / \mathrm{s}^{2}
$$

This is considerably smaller than the MOND optimized minimum acceleration of approximately $1.2 \times 10^{-10} \mathrm{~m} / \mathrm{s}^{2}$. However, the mathematical form of the MOND theory is different from what we are suggesting here; observational data is needed to make them directly comparable. First of all, our minimum acceleration is at the very edge of the observable universe. If the observations are concerning objects, such as galaxies, that are not at the edge of the universe, then the minimum acceleration could be higher. We will suggest the acceleration in the galaxy arms should be

${ }^{1}$ We suggested this first in a working paper that we put out on vixra.org March 29, 2020, this is a strongly improved version of that working paper. 


$$
a=\frac{G M}{r^{2}}+g_{\min }=\frac{G M}{r^{2}}+\frac{G M_{u}}{r_{u}^{2}}
$$

where $M$ is the baryonic matter in the galaxy, and $M_{u}$ is the mass of the universe, as before. In the next section, we will compare the prediction of this model with the observed data.

\section{Comparison of Our Model with Observational Data}

To test the model, we have used 2793 individual data points from 153 galaxies in the Spitzer Photometry and Accurate Rotation Curves (SPARC) database (see also [12]). Figure 1 shows the observations as black dots. The green line is the predicted galaxy rotation from only baryonic matter in the galaxy. As we see, the green line gives predictions far from the observed data, and this is why, as noted previously, the idea of dark matter was introduced originally in order to make this model work. The MOND best fit model is represented by the yellow line. The light blue line just below the yellow line is predictions from the quantized inertia model. The red line is our model when using radius $4.4 \times 10^{26} \mathrm{~m}$. As we

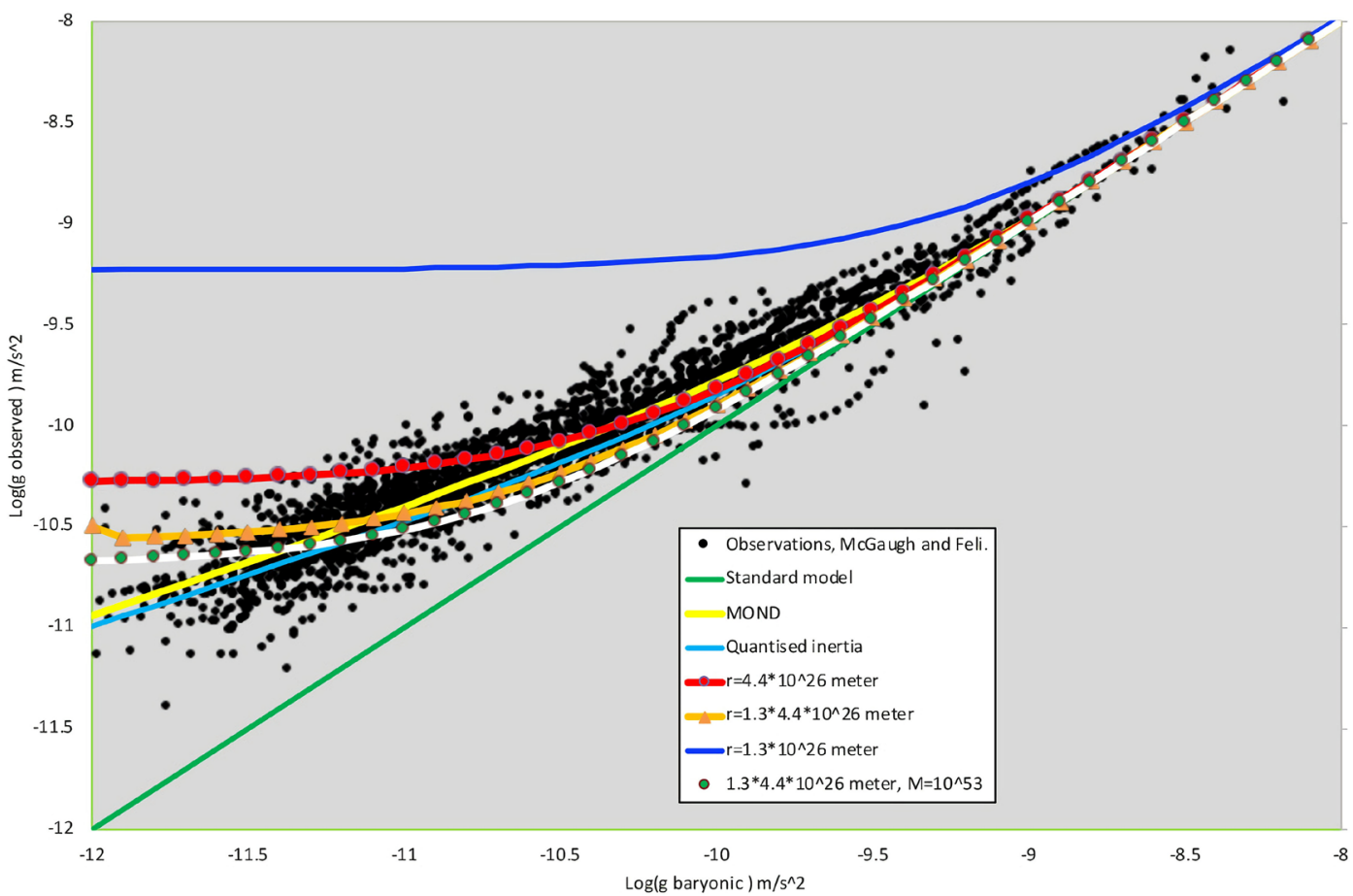

Figure 1. Galactic accelerations from 2793 individual data points for 153 SPARC galaxies are shown as black dots. Predictions by standard physics are shown in green. The yellow line is MOND, which fits the observations very well. The light blue line just below the yellow line indicates predictions made by the quantized inertia model. The red line includes the minimum acceleration from the mass in the observable universe with the standard assumed radius of approximately $4.4 \times 10^{26}$ meters. The orange line shows the results when we have multiplied this radius by 1.3. The white line with green dots shows the results when we have multiplied the universe radius by 1.3 , and the universe mass is adjusted $M_{u}=10^{53} \mathrm{~kg}$, compared to $M_{u}=1.5 \times 10^{53} \mathrm{~kg}$ in the red, orange and blue line. As we can see this simple model also can give predictions close to the observed data. The blue line depicts the predictions when we use a radius equal to the assumed time since the Big Bang multiplied by the speed of light. (Note: Log stands for Logarithm with base 10.) 
can see, this gives a strong improvement over the standard model, e.g., the green line. This would, at least, dramatically reduce the amount of dark matter required to push the model to fit observations. However, even under the standard model, it is not certain what the radius and the mass of the universe [13] [14] are, or what the distribution of matter is. Hence, we could suggest a slightly different radius, which would give an even better fit. In the case of the orange line, we have inputted a radius of 1.3 times the commonly assumed radius of $4.4 \times 10^{26} \mathrm{~m}$. The blue line shows the results when using only $1.3 \times 10^{26} \mathrm{~m}$ as radius. That is the radius one obtains by taking the assumed life of the universe times the speed of light; in other words, by ignoring the assumed expansion. This last value of $R$ we see gives predictions that diverge greatly from observations.

As we can clearly see, taking the mass of the observable universe into account, in addition to that of the galaxy, provides much better predictions than can be produced without doing so. However, there are several issues with this method. For example, if a Galaxy is lying at the edge of the observable universe, then the observable universe gravitational acceleration field should not only increase the acceleration in galaxy arms that are turned away from the centre of the observable universe, but should perhaps also slow the acceleration in the galaxy arms on the opposite side. This should lead to different redshifts on different sides of the galaxy. We do not believe this has been observed (at least not yet), but it could be even more complicated than this. Naturally, different galaxies will have different radii to the centre of the observable universe, so if we are taking this into account, we would possibly obtain a much better fit than what we have shown here. Or, counterintuitively, the fit could be worse; this can only be determined by further studies.

\section{Conclusion}

We have looked at galaxy rotation predictions when taking the gravity acceleration field from the observable universe into account. This seems to produce predictions quite close to observations. However, there may be several issues with this method and the approach requires additional rigorous study. Still, we think the idea is interesting and merits further investigation by the physics community.

\section{Conflicts of Interest}

The author declares no conflicts of interest regarding the publication of this paper.

\section{References}

[1] Newton, I. (1686) Philosophiae Naturalis Principia Mathematica. London. https://doi.org/10.5479/sil.52126.39088015628399

[2] Einstein, A. (1916) Näherungsweise integration der feldgleichungen der gravitation. Sitzungsberichte der Königlich Preussischen Akademie der Wissenschaften Berlin.

[3] Milgrom, M. (1983) A Modification of the Newtonian Dynamics as a Possible Alternative to the Hidden Mass Hypothesis. Astrophysical Journal, 270, 365-370. 
https://doi.org/10.1086/161130

[4] Corda, C. (2009) Interferometric Detection of Gravitational Waves: The Definitive Test for General Relativity. International Journal of Modern Physics D, 180, 2275-2282. https://doi.org/10.1142/S0218271809015904

[5] Bertolami, O., Böhme, C.G., Harko, T. and Lobo, F.S.N. (2007) Extra Force in $f(R)$ Modified Theories of Gravity. Physical Review D, 75, Article ID 104016. https://doi.org/10.1103/PhysRevD.75.104016

[6] McCulloch, M.E. (2017) Galaxy Rotations from Quantised Inertia and Visible Matter Only. Astrophys Space Science, 362, Article Number: 149. https://doi.org/10.1007/s10509-017-3128-6

[7] Bars, I. and Terning, J. (2009) Extra Dimensions in Space and Time. Springer, Berlin. https://doi.org/10.1007/978-0-387-77638-5

[8] Luque, B. and Ballesteros, F.J. (2019) To the Sun and beyond. Nature Physics, 15, 1302. https://doi.org/10.1038/s41567-019-0685-3

[9] Weinberg, S. (1972) Gravitation and Cosmology. Wiley, New York.

[10] Sivaram, C. (1982) Towards a Unification of the Parameters Underlying Elementary Particles and Cosmology. Astrophysics and Space Science, 88, 507. https://doi.org/10.1007/BF01092717

[11] Carvalho, J.C. (1995) Derivation of the Mass of the Observable Universe. International Journal of Theoretical Physics, 34, 2507. https://doi.org/10.1007/BF00670782

[12] McGaugh, S.S., Lelli, F. and Schombert, J.M. (2016) The Radial Acceleration Relation in Rotationally Supported Galaxies. Physical Review Letters, 117, Article ID 201101. https://doi.org/10.1103/PhysRevLett.117.201101

[13] Sitter, De.W. (1932) The Size of the Universe. Publications of the Astronomical Society of the Pacific, 44, 89. https://doi.org/10.1086/124203

[14] Whitrow, G.J. (1946) The Mass of the Universe. Nature, 159, 65. https://doi.org/10.1038/158165b0 